\title{
A Novel Circuit Model of Nanotechnology-Enabled Inkjet-Printed Gas Sensors Using Multi-Wall Carbon Nanotubes
}

\author{
Rosa De Paolis ${ }^{1,2,3}$, Taoran Le ${ }^{4}$, Fabio Coccetti ${ }^{1,2}$, Giuseppina Monti ${ }^{3}$, Luciano Tarricone ${ }^{3}$, Manos M. \\ Tentzeris $^{4}$, Robert Plana ${ }^{1,2}$ \\ ${ }^{1}$ CNRS; LAAS, 7 avenue du colonel Roche, F-31077 Toulouse, France \\ ${ }^{2}$ Université de Toulouse; UPS, INSA, INP, ISAE; LAAS, F-31077 Toulouse, France \\ ${ }^{3}$ University of Salento, Lecce, 73100, Italy \\ ${ }^{4}$ GEDC, School of ECE, Georgia Institute of Technology, Atlanta, GA 30332, U.S.A.
}

\begin{abstract}
This paper presents a novel electrical model of multi-walled carbon-nanotube based gas sensors completely patterned using low-cost inkjet printing. The obtained results (validated through measurements from $50 \mathrm{MHz}$ to $3 \mathrm{GHz}$ ) demonstrate that the nanostructure-based sensing mechanisms yields a quite evident shift of circuit resistive elements. A narrowband investigation of the resistive variation depending on gas concentration has been performed as well. The input impedance has been found to be significantly lower than that reported for metal oxide sensors, thus facilitating the integration in electronic circuitry. The suggested equivalent model can be exploited in the implementation of large-scale nanotechnologyenabled inkjet-printed modules.

Index Terms - Gas sensing, carbon nanotube, inkjet printing, equivalent circuit, nanotechnology.
\end{abstract}

\section{INTRODUCTION}

Due to their excellent mechanical and electronic properties, carbon nanotubes (CNTs) are attractive nanoscale materials which have been gaining great interest in a large variety of applications of microelectronics and nanotechnologies. In particular, very promising gas sensing capabilities at room temperature have been demonstrated due to their hollow core, large surface area, and small size. More specifically, chemical absorption produces changes in electrical properties (such as impedance and effective permittivity), that can be exploited to determine chemical compounds and concentration $[1,2]$.

In the past it has been demonstrated that CNTs can be successfully deposited by inkjet printing and used in the fabrication of tunable microwave circuits, such as resonators, antennas, and RFID tags [3]. Furthermore, the possibility to print CNT-ink based gas sensors on cheap paper substrates has been displayed as well $[4,5]$. Nevertheless, up to now only few studies about modeling of CNTs have been reported, mainly concerning a CNT-ink not sensing structure [6] or a not printed CNT sensing element [7].

In this paper, a novel electrical circuit model has been accomplished for a gas sensor whose structure (including both sensing element and access lines) has been completely patterned using low-cost inkjet printing on paper substrate. The sensing section consists in water soluble multi-walled carbon-nanotube (MWCNT) that has been patterned within two silver-nanoparticle based microstrip transmission lines. The proposed model represents a comprehensive electrical description of the sensing mechanism and it has been validated through frequency measurements from $50 \mathrm{MHz}$ to 3 $\mathrm{GHz}$, showing that, under $\mathrm{NO}_{2}$ exposure at room temperature, a shift of the circuit resistive elements is produced. Furthermore, the resistive variation as a function of gas concentration has been investigated by means of two narrowband analyses performed around $866.5 \mathrm{MHz}$ and $2.4 \mathrm{GHz}$ (corresponding to European UHF RFID center frequency and WiFi band, respectively). In these bands, the impedance magnitude associated to the MWCNT sensor is below $0.5 \mathrm{k} \Omega$, that is significantly lower than that reported for metal oxide sensors $(0.5 \mathrm{M} \Omega-50 \mathrm{M} \Omega)$ [2], thus facilitating the integration in more complex circuits. In addition, the suggested equivalent model, that combines the effects of nanostructures, gas absorption/desorption macroscopic effect and inkjet printing up to the wireless frequencies, would enable the large scale implementation of RF nanotechnologybased inkjet-printed modules. For instance, this approach could allow for very high-efficiency chip-less passive RFIDenabled gas sensors by means of a very accurate matching between the antenna and the gas-sensitive load (critical aspect for the reading range and the backscattered power in RFID systems).

\section{StRuCTURE DESCRIPTION AND EQUiVAlENT CIRCUIT}

For proof of concept and without loss of generality, test samples have been fabricated onto low-cost paper substrate and consist of an inkjet printed MWCNT ink-based strip deposited in the $1 \mathrm{~mm}$ gap separating two inkjet printed silvernanoparticle based $2 \mathrm{~mm}$-width microstrip transmission lines (Fig. 1). Both silver ink and MWCNT ink have been deposited via a Dimatix DMCLCP-11610 printer using $10 \mathrm{pL}$ cartridges.

As for the MWCNT ink, it has been produced via chemical vapor deposition, purified with acid, and functionalized to improve in water solubility. Glycol is added to increase the viscosity of the material and enhance flow through the printer. 
Immediately prior to starting the printing process, the mixture is sonicated for 1 hour and degassed for the same duration.

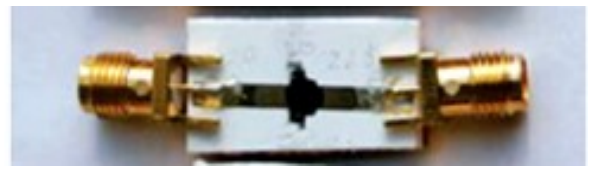

Fig. 1. Test sample: inkjet printed MWCNT strip deposited in the gap separating two inkjet printed silver-nanoparticle microstrip transmission lines [5].

Frequency measurements of the test samples have been taken by vector network analyzer (R\&S ZVA8) both in air and in the presence of different $\mathrm{NO}_{2}$ concentrations, thus allowing discriminating the gas contribution on the MWCNT electrical properties. More specifically, the effect of gas has been examined at room temperature by using a TIN-TEK FlexStream gas standards generator, providing an extremely stable and accurate mix of gas at extremely low concentrations $[5]$.

A preliminary test on the sensitivity of this structure to $\mathrm{NO}_{2}$ (defined as the change of the material's impedance in presence of the test gas relative to air, over that of air) has been carried out in [5], where it has been investigated as a function of both gas concentration and exposure time at just two frequency points $(864 \mathrm{MHz}$ and $2.4 \mathrm{GHz}$ ). This test demonstrated that at these frequencies the MWCNT ink has high sensitivity and a very fast response. Consequently, for the experiment reported in this work it has been chosen to take measurements after 1 minute of exposure.

In the present work, the behavior of the same sample has been thoroughly investigated in a broader frequency range, i.e. from $50 \mathrm{MHz}$ to $3 \mathrm{GHz}$. At first, an equivalent circuit has been obtained by semi-empirical approach for the "in air" (no gas presence) condition. Afterwards, the reaction mechanisms introduced by MWCNTs by exposure to $\mathrm{NO}_{2}$ at different concentrations have been associated to electrical properties by means of variable elements in the equivalent circuit.

Fig. 2 shows the "macroscopic" equivalent circuit in air; the test sample can be modeled by means of two microstrip transmission lines (TLs) for the input and output sections, and by lumped elements for the core sensing section. More specifically, the core section of the equivalent circuit takes into account both the inkjet printed MWCNTs' sensing strip at the gap region and the conductor ink/CNT ink overlapping contact areas. Furthermore, based on the results reported in [3], the properties of the paper substrate have been also considered.

With respect to the models present in the literature (such as the one in [7]), the equivalent circuit here proposed uses a greater number of parameters, thus allowing a more accurate and a more broadband description of the sensor structure.

\section{RESULTS AND DISCUSSION}

The lumped elements of the equivalent circuit "in air" have been optimized to obtain the best fitting result between measurements and simulations over the entire frequency range (from $50 \mathrm{MHz}$ to $3 \mathrm{GHz}$ ). In particular, impedance parameters have been obtained through S-parameters conversion performed by vector network analyzer (R\&S ZVA8). Comparisons between simulation results of the proposed equivalent model and impedance measurements in air are shown in Fig. 3. A good agreement has been achieved.

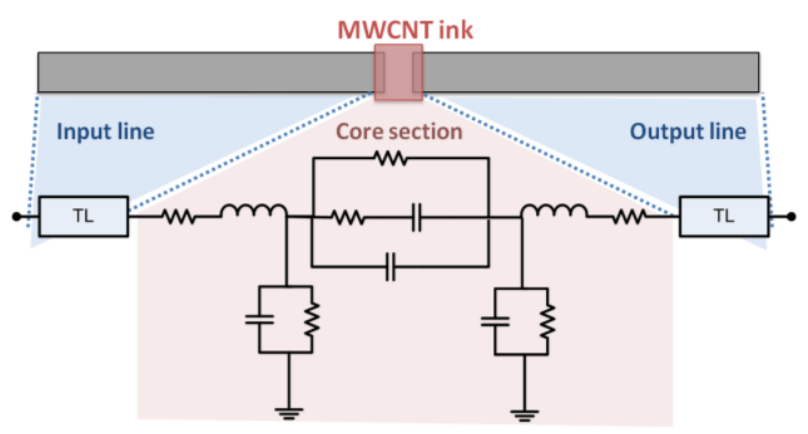

Fig. 2. Equivalent circuit of gas sensing structure in air.

As for the extracted parameters, capacitance values are between 2 and $10 \mathrm{pF}$, resistive values are between 8 and $130 \Omega$, and inductance is about $0.4 \mathrm{nH}$.

When the test sample is exposed to $\mathrm{NO}_{2}$ gas, a change of the MWCNT material properties is taking place, as verified by impedance measurements, and it can be associated to variable elements in the equivalent circuit.
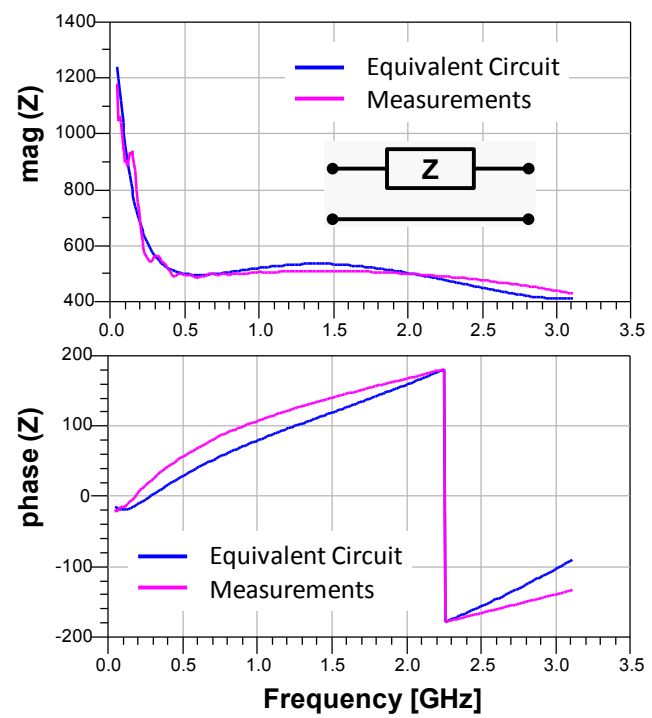

Fig. 3. Comparisons between simulation on the proposed equivalent circuit and impedance measurements in air. 
The proper choice of circuit elements affected by gas exposure is not straightforward and univocal. By means of a sensitivity analysis, the most sensitive parameters have been found to be the resistances in Fig. 4. Consequently, they will be considered as the variable elements associated to sensing mechanism. This resistive response can also be seen as the superposition of the effects of gas absorption on the contact resistance and on the MWCNT resistance, as confirmed in [9].

The electrical resistances were found to decrease under $\mathrm{NO}_{2}$ gas. This result is supported by the literature $[1,2,8]$, where the interpretation of the electrical response of CNT has been explained by considering the chemical nature of the $\mathrm{NO}_{2}$ molecule. In particular, $\mathrm{NO}_{2}$ has an unpaired electron and is known as a strong oxidizer, thus meaning that, upon gas adsorption, a charge transfer occurs from the CNTs to the $\mathrm{NO}_{2}$ that increases hole carriers in CNT and reduces resistance.

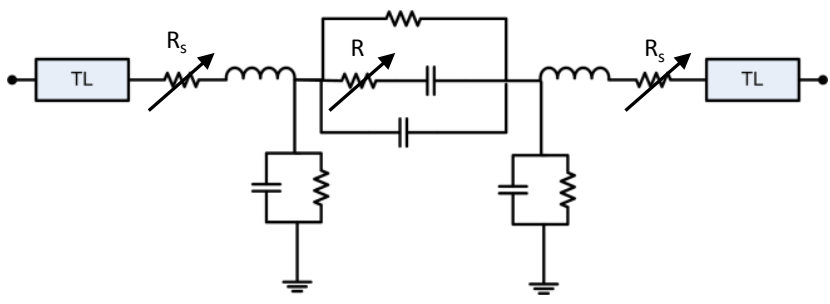

Fig. 4. Equivalent circuit and variable elements under $\mathrm{NO}_{2}$ gas exposure.

The parameters deviation from the "in air" condition as a function of the gas concentration has been investigated as well. For this purpose a narrowband analysis has been performed around two different frequencies, i.e. $866.5 \mathrm{MHz}$ and $2.4 \mathrm{GHz}$. The most prominent variation effects can be observed by comparing (10ppm, 30ppm, 90ppm) gas concentration. The results show that by increasing the gas concentration the values of the resistive components decrease even further. In addition, it is worth noting that the magnitude of the input impedance of the MWCNT sensor (extracted by measurements) is below $0.5 \mathrm{k} \Omega$ in both of the frequency bands of interest. This value is significantly lower than that reported in [2] for metal oxide sensors $(0.5 \mathrm{M} \Omega-50 \mathrm{M} \Omega)$, thus facilitating the integration of this sensor structure in conventional RF and RFID circuits.

The comparisons between simulation results performed on the proposed equivalent model and impedance measurements (in magnitude) in air and at different $\mathrm{NO}_{2}$ concentrations are shown in Fig. 5 (fitting results on the phase do not provide further useful information and are not shown here).

A study of the resistance variation as a function of the gas concentration values has been performed as well. More specifically, the variation of the resistances $R$ and $R_{s}$ with respect to their "in air" condition has been extracted at $866.5 \mathrm{MHz}$ and at $2.4 \mathrm{GHz}$ by means of the following formula:

$$
\Delta R_{x}=100 *\left(R_{\text {air }}-R_{\text {gas }}\right) / R_{\text {air }}
$$

where $R_{\text {air }}$ is the value of $\mathrm{R}$ and $\mathrm{R}_{\mathrm{s}}$ in air, while $R_{\text {gas }}$ is the value of $R$ and $R_{s}$ in different gas concentrations.
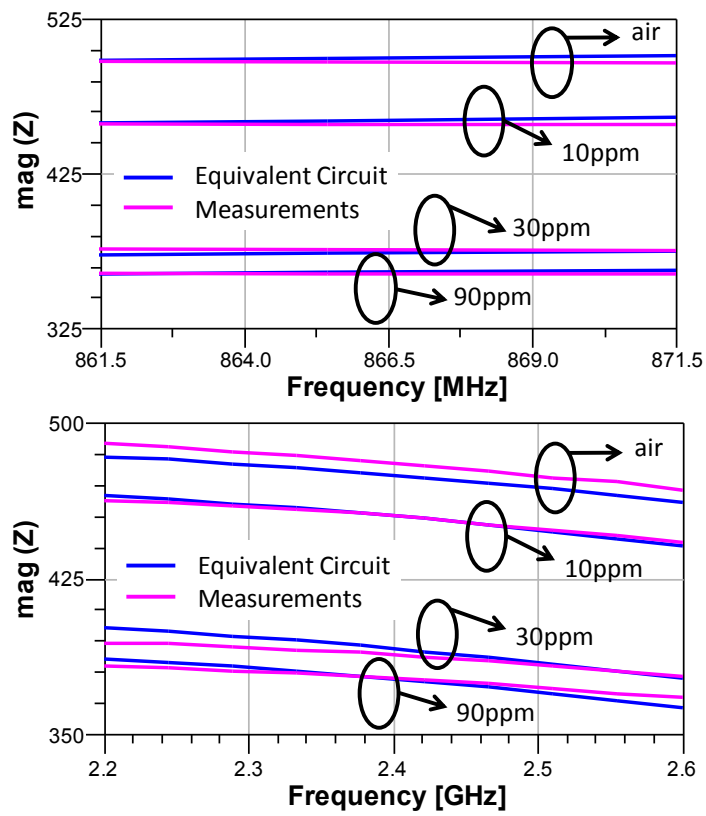

Fig. 5. Narrowband fitting around $866.5 \mathrm{MHz}$ and $2.4 \mathrm{GHz}$ : comparison between simulation on the proposed equivalent circuit and impedance measurements in $\mathrm{NO}_{2}$ at different concentrations.

The behavior of $\Delta R_{x}$ as a function of $\mathrm{NO}_{2}$ gas concentration has been analyzed at two different frequencies $(866.5 \mathrm{MHz}$ and $2.4 \mathrm{GHz}$ ) and is reported in Fig. 6. The results show that at both frequencies the gas exposure affects the resistance associated to MWCNT region (R) in a much greater degree than the resistance of the contact region $\left(R_{s}\right)$, and that both $R$ and $\mathrm{R}_{\mathrm{s}}$ have a most prominent variation at $866.5 \mathrm{MHz}$ than at $2.4 \mathrm{GHz}$.

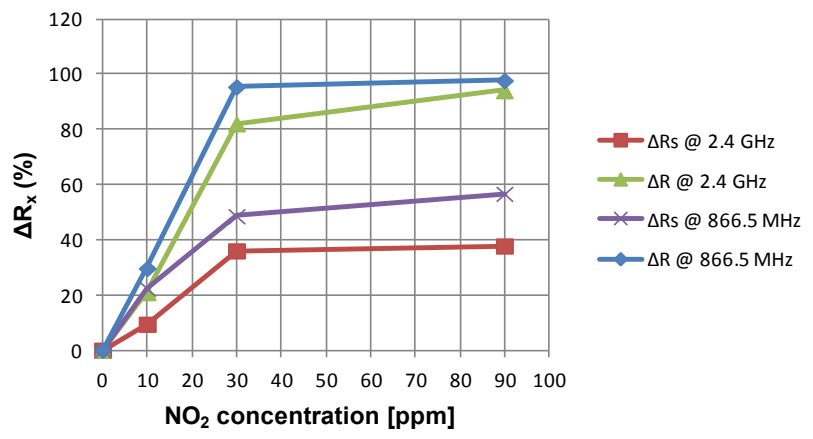

Fig. 6. The behavior of the resistive variation (with respect to the "in air" condition) as a function of the gas concentration.

Furthermore, the variation of both $\mathrm{R}$ and $\mathrm{R}_{\mathrm{s}}$ seems to follow a linear behavior up to $30 \mathrm{ppm}$ of gas, while at upper concentration values it nearly becomes constant. This saturation mechanism could be due to the fact that all CNTs have reacted. If we approximate the behavior seen up to $30 \mathrm{ppm}$ with a linear equation, the resistance $\mathrm{R}$ increases with gas concentration by following a slope of 2.7 at $2.4 \mathrm{GHz}$ and 
of 3.1 at $866.5 \mathrm{MHz}$, whereas the curve of the resistance $\mathrm{R}_{\mathrm{s}}$ has a slope of 1.2 at $2.4 \mathrm{GHz}$ and of 1.7 at $866.5 \mathrm{MHz}$, thus confirming the most prominent variation of both resistances at the lower frequency of analysis.

\section{CONCLUSION}

An electrical model of multi-walled carbon-nanotube based gas sensors totally patterned using low-cost inkjet transfer printing has been presented. The test sample is a high sensitive gas sensor printed on cheap paper substrate and exposed to different concentrations of nitrogen dioxide. Impedance measurements from $50 \mathrm{MHz}$ to $3 \mathrm{GHz}$ at room temperature have been performed in order to validate the equivalent circuit.

The proposed equivalent model provides a comprehensive description of MWCNT inkjet printed gas sensor up to the wireless frequencies, including both nanostructures, and gas absorption/desorption macroscopic effects.

Results demonstrate that the sensing mechanisms introduced by gas can be mainly related to a resistive variation in the equivalent circuit (associated to the contact resistance and to the MWCNT resistance).

Furthermore, the resistive variation as a function of gas concentration has been investigated by means of narrowband analyses. The achieved results demonstrate that the gas exposure mainly affects the resistance associated to MWCNT region compared to the resistance of the MWCNT/silver contact region. The behavior of the resistive variation has been confirmed at two different frequencies $(866.5 \mathrm{MHz}$ and $2.4 \mathrm{GHz}$ ). Last, but not least, in the two frequency ranges of interest, the measured impedance magnitude is below $0.5 \mathrm{k} \Omega$, i.e. significantly lower than that of metal oxide sensors, thus facilitating the integration of the MWCNT ink based sensors in electronic circuits.

Reported results confirm that MWCNT ink is a really promising candidate for gas detection and that the suggested equivalent model can be exploited in the large scale implementation of RF nanotechnology-based inkjet-printed modules, including high-efficiency chip-less passive RFIDenabled sensor networks. In such a context, this approach allows a very accurate matching between the antenna and the gas-sensitive load, which is a common critical aspect for the reading range and the backscattered power in RFID systems.

\section{REFERENCES}

[1] J. Kong, N. R. Franklin, C. Zhou, M. G. Chapline, S. Peng, K. Cho, H. Dai, "Nanotube Molecular Wires as Chemical Sensors," Science, vol. 287, pp. 622-625, January 2000.

[2] L. Valentini, I. Armentano, J. M. Kenny, C. Cantalini, L. Lozzi, S. Santucci, "Sensors for sub-ppm $\mathrm{NO}_{2}$ gas detection based on carbon nanotube thin films," Appl. Phys. Lett., vol. 82, no. 6, pp. 961-963, 2003.

[3] L. Yang, A. Rida, R. Vyas, and M.M. Tentzeris, "RFID tag and RF structures on paper substrates using inkjet-printing technology," IEEE Trans. on Microwave Theory \& Tech., vol. 55, no.12, pp. 2894-2901, December 2007.

[4] L. Yang, R. Zhang, D. Staiculescu, C.P. Wong, and M.M. Tentzeris, "A novel conformal RFID-enabled module utilizing inkjet-printed antennas and carbon nanotubes for gas-detection applications," IEEE Antennas and Wireless Propagation Letters, vol. 8, pp. 653-656, June 2009.

[5] V. Lakafosis, X. Yi, T. Le, E. Gebara, Y. Wang, and M. M. Tentzeris, "Wireless Sensing with Smart Skins," IEEE Sensors, pp. 623-626, 2011.

[6] R. De Paolis, S. Pacchini, F. Coccetti, G. Monti, L. Tarricone, M. M. Tentzeris, and R. Plana, "Circuit Model of CarbonNanotube Inks for Microelectronic and Microwave Tunable Devices ," IEEE MTT International Microwave Symposium Digest, 5-10 June, 2011.

[7] O.K. Varghese, P.D. Kichambre, D. Gong, K.G. Ong, E.C. Dickey, C.A. Grimes, "Gas sensing characteristics of multi-wall carbon nanotubes," Sensors and Actuators B: Chemical, vol. 81, no. 1, pp. 32-41, December 2001.

[8] S. Rajaputra, R. Mangu, P. Clore, D. Qian, R. Andrews, and V. P Singh, "Multi-walled carbon nanotube arrays for gas sensing applications," Nanotechnology, vol. 19, 2008.

[9] J. Suehiro, H. Imakiire, S. Hidaka, W. Ding, G. Zhou, K. Imasaka, M. Hara, "Schottky-type response of carbon nanotube $\mathrm{NO}_{2}$ gas sensor fabricated onto aluminum electrodes by dielectrophoresis," Sensors and Actuators B, vol. 114, pp. 943949, 2006. 